\title{
PROPRIEDADES SENSORIAIS E FÍSICO-QUÍMICAS DE LEITES FERMENTADOS COMERCIAIS
}

\section{SENSORIAL AND PHYSICAL-CHEMICAL PROPERTIES OF COMMERCIAL FERMENTED MILK}

\author{
Maike Taís Maziero ${ }^{1}$ Marina Caldeira Tolentino ${ }^{1}$; Nina Waszczynskyj ${ }^{1}$ \\ Universidade Federal do Paraná - PPGTA - Curitiba - Brasil maikemaziero@ yahoo.com.br
}

\begin{abstract}
Resumo
O consumo de leites fermentados está em ascensão devido ao apelo por suas propriedades funcionais e a diversidade de marcas disponíveis no mercado aumenta proporcionalmente. $O$ presente trabalho teve como objetivo avaliar as propriedades sensoriais e físico-químicas de três marcas disponíveis no mercado brasileiro. Foram realizadas análises físico-químicas de acidez, pH, viscosidade, teor de sólidos totais e solúveis e as avaliações sensoriais de perfil de atributos e ordenação por preferência com provadores não treinados. Não houve diferença significativa entre as marcas avaliadas para nenhum dos atributos sensoriais, apesar de terem apresentado diferenças significativas nos parâmetros físico-químicos de acidez, viscosidade e sólidos solúveis e totais.
\end{abstract}

Palavras-chave: Lactobacillus casei, probióticos, perfil de atributos.

\section{Introdução}

O Regulamento Técnico de Identidade e Qualidade descrito pelo Ministério da Agricultura, Pecuária e Abastecimento define leites fermentados como produtos adicionados ou não de outras substâncias alimentícias, obtidas por coagulação e diminuição do $\mathrm{pH}$ do leite, ou reconstituído, adicionado ou não de outros produtos lácteos cuja fermentação se realiza com um ou vários dos seguintes cultivos: Lactobacillus acidophilus, Lactobacillus casei, Bifidobacterium sp, Streptococus salivarius subsp thermophilus e/ou outras bactérias acido-lácticas que, por sua atividade, contribuem para a determinação das características do produto final (BRASIL, 2007).

O processo fermentativo modifica as propriedades sensoriais e o valor nutritivo do leite, prolonga seu prazo comercial e amplia a gama de produtos lácteos (ORDOÑEZ, 2005).

Produtos lácteos contendo culturas probióticas como Lactobacillus casei apresentam vários benefícios à saúde, uma vez que aliviam a intolerância à lactose, inibem a adesão de microrganismos patogênicos à mucosa intestinal prevenindo a diarréia e estimulam o sistema imune 
(VINDEROLA et al., 2000). Estes microrganismos fermentam a lactose, dando origem ao ácido lático e a outros ácidos orgânicos, como o acético e o propiônico. Portanto, o leite fermentado normalmente é ácido, com pH variando entre 3,5 e 4,5. A porcentagem de ácido lático deve ser de, no mínimo, 0,60\% para garantir a estabilidade microbiológica do produto (FARIA et al., 2006).

O primeiro leite fermentado com Lactobacillus casei chegou ao Brasil em 1966 e, em 2001, foi aprovado pela ANVISA (Agência Nacional de Vigilância Sanitária) como o primeiro leite fermentado com alegações de propriedades funcionais e/ou de saúde. Nos últimos anos, várias marcas de leites fermentados têm surgido no mercado nacional, aumentando a oferta deste produto e tornando-os mais acessíveis ao consumidor.

O presente trabalho teve como objetivo avaliar o perfil de características sensoriais e físicoquímicas de três amostras comerciais de leites fermentados.

\section{Material e Métodos}

As amostras de leite fermentado foram adquiridas no comércio de Curitiba, Paraná, Brasil, em julho de 2009 e armazenadas sob refrigeração $\left(4{ }^{\circ} \mathrm{C}\right)$ até o momento das análises, sendo duas marcas tradicionais no mercado nacional (identificadas como marca A e B), e a terceira introduzida recentemente (marca $\mathrm{C})$.

As análises físico-químicas das três marcas de leite fermentado foram feitas em triplicata. A determinação do teor de sólidos solúveis ( ${ }^{\circ}$ Brix) foi realizada em refratômetro modelo Abbé 2WAJ; o teor de sólidos totais em balança de infravermelho modelo Gehaka BG200; o pH em pHmetro modelo Mezuro Píon-310 e a viscosidade cinemática em Viscosímetro Tecnal com capilar 150 (CECCHI, 2003).

A determinação da acidez foi realizada através de titulação com solução de hidróxido de sódio $0,1 \mathrm{~N}$, de acordo com a Instrução Normativa 68 , que estabelece os métodos analíticos oficiais físico-químicos para controle de leite e produtos lácteos (BRASIL, 2006).

A análise sensorial foi realizada no Laboratório de Análise Sensorial do Departamento de Tecnologia de Alimentos da Universidade Federal do Paraná (UFPR), com trinta e três estudantes universitários e funcionários de ambos os sexos e com idades entre 19 e 69 anos. As amostras foram dispostas aos julgadores de forma aleatória, em copos com capacidade para $50 \mathrm{~mL}$, contendo $30 \mathrm{~mL}$ de leite fermentado e codificados com números de três dígitos, obtidos de uma tabela de números aleatórios. Foi fornecido biscoito de "água e sal” e água para que os julgadores pudessem realizar a limpeza do palato entre uma amostra e outra.

Para avaliação da preferência foram realizados os métodos de perfil de atributos de acordo com a NBR 14141 e teste de ordenação conforme NBR 13170 (ABNT, 1998; ABNT, 1994). No 
perfil de atributos, foram avaliados sabor, aroma, viscosidade e aparência pela aplicação de uma escala hedônica, cujos extremos correspondiam a desgostei muitíssimo (1) e gostei muitíssimo (9). Foi solicitado aos julgadores que analisassem cada amostra e classificassem os atributos de acordo com a escala proposta. Os resultados foram tratados estatisticamente, realizando análise de comparação de médias e análise de agrupamento, utilizando os programas Microsoft Excel 2007 e Statistica versão 7.0. No teste de ordenação, foi solicitado aos julgadores que classificassem a amostra de acordo com a preferência. Os resultados foram avaliados pelo teste de Friedman utilizando tabela de Newel e MacFarlane a 5\% de significância.

\section{Resultados e Discussão}

Os resultados das análises físico-químicas são apresentados na Tabela 1.

Tabela 1- Resultados das análises físico-químicas das três marcas comerciais de leite fermentado

\begin{tabular}{lccc}
\hline Análise & Marca A & Marca B & Marca C \\
\hline Acidez (\% ácido lático) & $1,04 \pm 0,020^{\mathrm{b}}$ & $1,11 \pm 0,010^{\mathrm{b}}$ & $1,32 \pm 0,010^{\mathrm{a}}$ \\
$\mathrm{pH}$ & $3,71 \pm 0,036^{\mathrm{a}}$ & $3,79 \pm 0,036^{\mathrm{a}}$ & $3,60 \pm 0,043^{\mathrm{a}}$ \\
Sólidos Solúveis ( ${ }^{\circ}$ Brix) & $18,00 \pm 0,433^{\mathrm{b}}$ & $14,50 \pm 0,250^{\mathrm{c}}$ & $20,00 \pm 0,629^{\mathrm{a}}$ \\
Sólidos Totais (\%) & $19,00 \pm 0,606^{\mathrm{b}}$ & $15,31 \pm 0,435^{\mathrm{c}}$ & $21,53 \pm 0,616^{\mathrm{a}}$ \\
Viscosidade cinemática (cSt) & $2,356 \pm 0,021^{\mathrm{b}}$ & $2,885 \pm 0,087^{\mathrm{b}}$ & $5,405 \pm 0,039^{\mathrm{a}}$ \\
\hline
\end{tabular}

* Médias com letras distintas em mesma linha diferem significativamente, com $95 \%$ de confiabilidade.

A acidez das três marcas avaliadas encontra-se de acordo com o Regulamento Técnico de Identidade e Qualidade de leites fermentados (BRASIL, 2007), que prevê a porcentagem de ácido lático entre 0,6 e 2,0. As marcas A e B não diferiram estatisticamente, no entanto a marca C apresentou acidez mais elevada. Estes resultados foram superiores aos relatados por Wang et al. (2009), que encontraram valores variando de 0,80 a $0,93 \%$ de acidez em leite bovino fermentado por Lactobacillus casei; os autores relataram um pequeno aumento na acidez durante os 28 dias do prazo comercial deste produto.

A acidez exerce grande influência sobre os atributos de qualidade dos produtos lácteos fermentados e é um dos fatores que limita sua aceitação. Durante a estocagem refrigerada das bebidas lácteas, pode haver aumento da acidez titulável. Estas mudanças na acidez do produto ocorrem, em maior ou menor grau, dependendo da temperatura de refrigeração, do tempo de armazenamento, do poder de pós-acidificação das culturas utilizadas e também se relaciona às mudanças nos valores de $\mathrm{pH}$ (THAMER e PENNA, 2006). 
Todas as marcas encontram-se dentro do limite de $\mathrm{pH}$ no qual o crescimento das bactérias lácticas desenvolve-se normalmente e sem prejuízo, ou seja, entre 3,6 a 4,3 (VEISSEYRE, 1988). As médias de $\mathrm{pH}$ variaram de 3,60 a 3,79 e não houve diferença estatística entre as marcas. Estes resultados foram similares aos encontrados por Granato (2008), que avaliou 11 amostras de leites fermentados comerciais e encontrou resultados variando de 3,51 a 4,21.

Houve correlação positiva significativa $(\mathrm{p}<0,05)$ entre o teor de sólidos solúveis ( ${ }^{\circ}$ Brix) e o teor de sólidos totais nas três marcas avaliadas, sendo que as três diferiram estatisticamente para estas duas análises. Thamer e Penna (2006) encontraram valores de sólidos totais variando de 15,68 a 18,97\% em bebidas lácteas funcionais fermentadas por probióticos. Os sólidos solúveis representam os compostos solúveis em água, tais como, açúcares, ácidos, vitaminas e algumas gomas que podem ser adicionadas aos produtos lácteos para conferir textura (OLIVEIRA e DAMIN, 2003). A viscosidade da amostra $\mathrm{C}$ foi significativamente superior à das amostras $\mathrm{A}$ e $\mathrm{B}$, fato que pode ser explicado pela presença de pectina na composição deste leite fermentado.

Os resultados do teste de preferência em relação aos atributos sabor, aroma, viscosidade e aparência são apresentados na Tabela 2.

Tabela 2 - Resultado do teste de preferência das três amostras de leite fermentado

\begin{tabular}{llll}
\hline Atributos & Marca A & Marca B & Marca C \\
\hline Sabor & $6,12 \pm 2,24^{\mathrm{a}}$ & $6,42 \pm 2,00^{\mathrm{a}}$ & $6,30 \pm 1,90^{\mathrm{a}}$ \\
Aroma & $6,55 \pm 2,03^{\mathrm{a}}$ & $6,52 \pm 1,79^{\mathrm{a}}$ & $6,73 \pm 1,61^{\mathrm{a}}$ \\
Viscosidade & $6,67 \pm 1,67^{\mathrm{a}}$ & $6,79 \pm 1,62^{\mathrm{a}}$ & $6,97 \pm 1,49^{\mathrm{a}}$ \\
Aparência & $7,00 \pm 1,58^{\mathrm{a}}$ & $7,00 \pm 1,48^{\mathrm{a}}$ & $7,00 \pm 1,27^{\mathrm{a}}$ \\
\hline
\end{tabular}

* Média seguidas da mesma letra não diferem significativamente entre si, a 5\% de significância; $n=33$.

Pode-se observar que não houve diferença significativa entre as três amostras em relação a todos os atributos. Mesmo com a diferença entre os atributos físico-químicos das amostras, isso não foi percebido pelos julgadores. A marca $\mathrm{A}$, mais tradicional no mercado, não mostrou destaque como esperado.

Como não houve diferença significativa entre as amostras, foi realizada uma análise de agrupamento (Figura 1) para verificar a similaridade entre as mesmas, levando em consideração todos os atributos analisados, de forma global.

Por meio da análise do gráfico é possível observar que há maior similaridade entre as amostras $\mathrm{B}$ e $\mathrm{C}$, do que entre a amostra $\mathrm{A}$ em relação às mesmas. Isso pode ocorrer pelo fato das amostras mais similares pertencem ao mesmo fabricante, com poucas diferenças em relação aos ingredientes declarados no rótulo. Por analogia, conclui-se que as marcas B e C foram 26,3\% mais similares entre si do que a marca A em relação ao agrupamento formado pelas amostras B e C. 
Figura 1 - Análise de agrupamento por distância euclidiana das três amostras comerciais

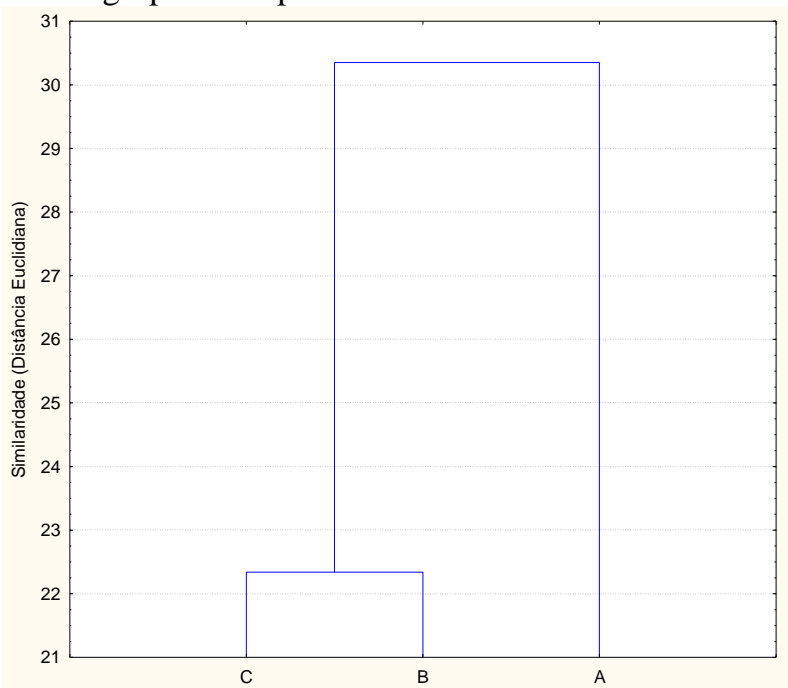

As freqüências das notas recebidas pelas três amostras para cada atributo no teste de preferência estão demonstradas nas Figuras 2, 3, 4 e 5.

Figura 2 - Frequência das notas recebidas pelas três amostras em relação ao atributo sabor

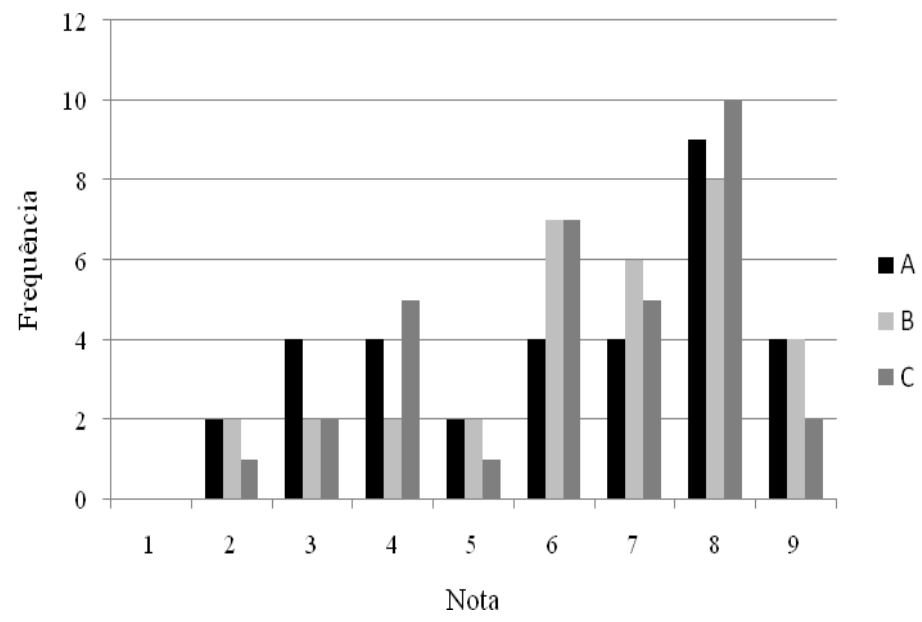

Figura 3 - Frequência das notas recebidas pelas três amostras em relação ao atributo aroma

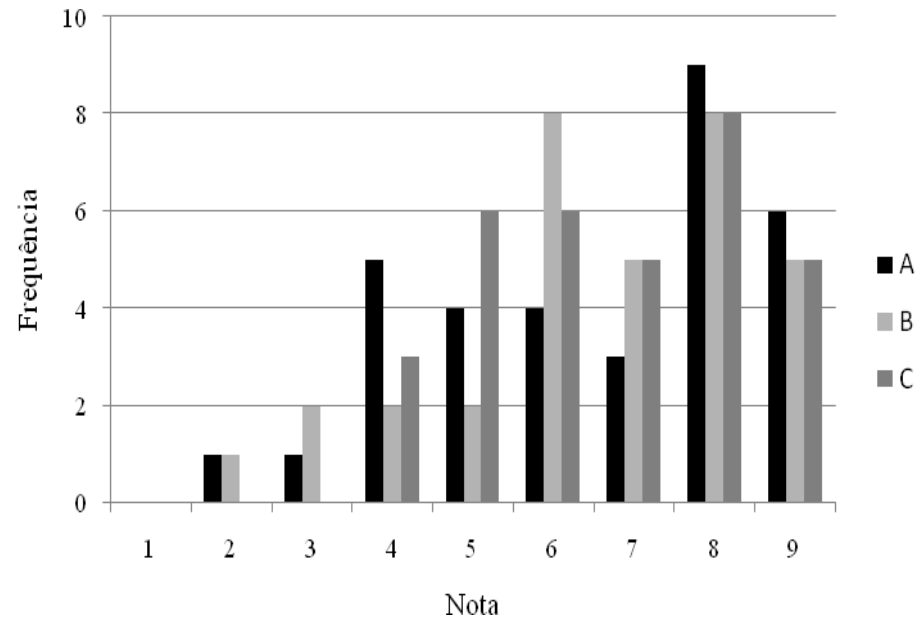


Figura 4 - Frequência das notas recebidas pelas três amostras em relação ao atributo viscosidade

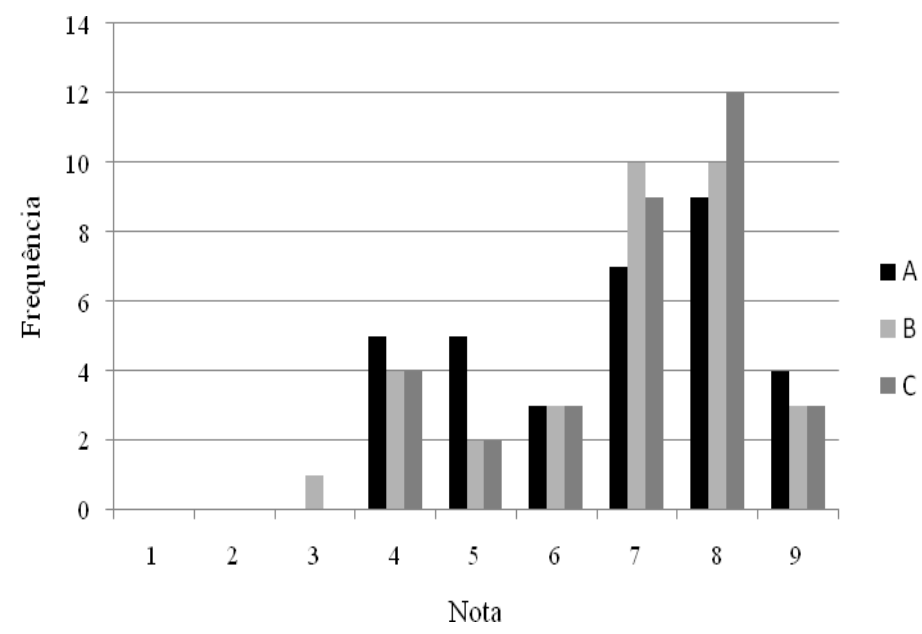

Figura 5 - Frequência das notas recebidas pelas três amostras em relação ao atributo aparência.

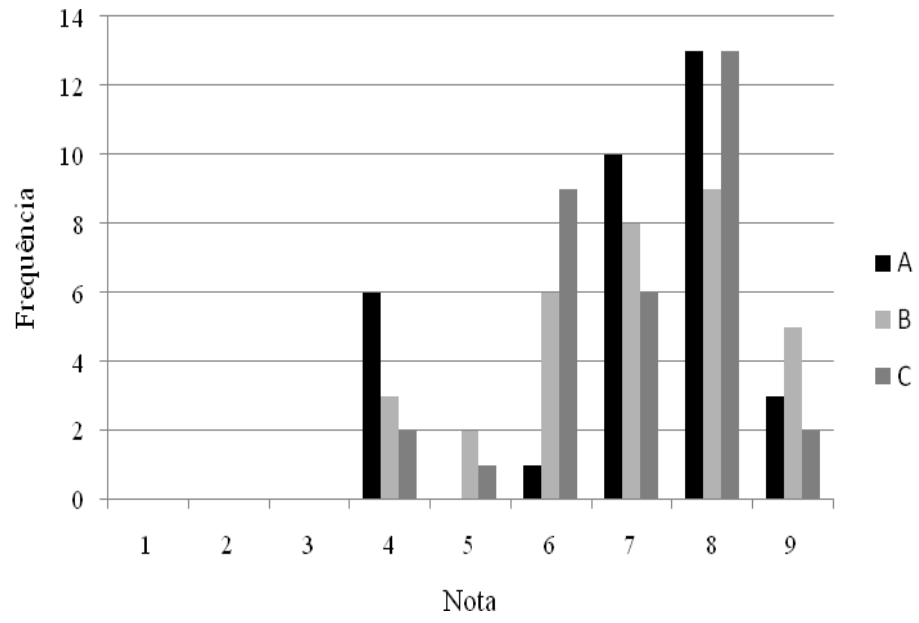

A partir da análise dos gráficos, pode-se observar que houve uma distribuição de notas muito semelhante entre as três amostras. Nenhuma amostra apresentou destaque.

Os resultados do teste de ordenação quanto à preferência são apresentados na Tabela 3.

Tabela 3 - Resultado do teste de ordenação quanto à preferência das três amostras de leite fermentado

\begin{tabular}{lccc}
\hline & Marca A & Marca B & Marca C \\
\hline Total & $68^{\mathrm{a}}$ & $67^{\mathrm{a}}$ & $63^{\mathrm{a}}$ \\
Diferença versus A & - & 1 & 5 \\
Diferença versus B & - & - & 4 \\
\hline
\end{tabular}

* Totais seguidos da mesma letra não diferem significativamente entre si, a 5\% de significância; DMS=20

De acordo com a Tabela de Newel e Mac Farlane, a diferença crítica entre os totais das somas de ordenação, a 5\% de significância, para o número de amostras e julgadores em questão é 20. Considerando que todos os valores de diferença são menores que o valor tabelado, conclui-se que não houve diferença significativa entre as amostras em relação à preferência. 
Na Figura 6 está apresentada a frequiência do posicionamento das amostras no teste de ordenação (1 - amostra preferida, 3 - amostra menos preferida).

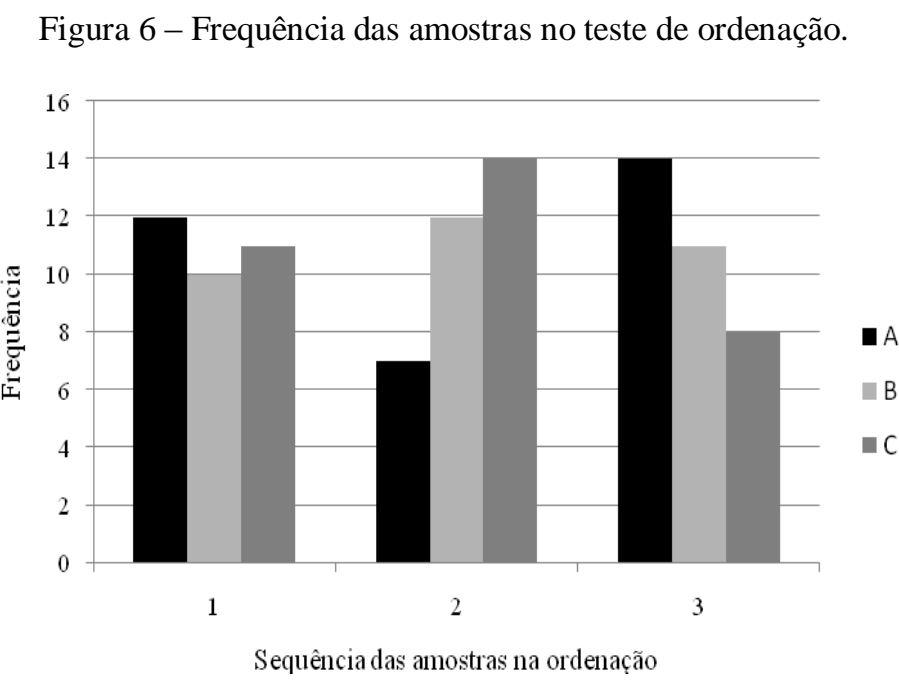

Fica comprovado a partir da análise da Figura 6 que há uma falta de tendência entre os julgadores no que diz respeito à preferência, uma vez que a amostra $\mathrm{A}$ foi a mais preferida $\mathrm{e}$ também a menos preferida. Isso porque recebeu um maior número de vezes a nota 1 e 3 quando comparada com as outras amostras.

\title{
4. Conclusões
}

Os leites fermentados são recomendados por suas propriedades funcionais e benefícios a saúde; devido a esses fatores seu consumo vem aumentando consideravelmente, paralelamente à maior diversidade na oferta de marcas disponíveis no mercado. Não houve diferença significativa entre as marcas deste estudo em nenhum dos atributos avaliados pela análise sensorial, apesar das amostras terem apresentado diferenças significativas em alguns parâmetros físico-químicos.

\begin{abstract}
The consumption of fermented milk is rising due to the resort for their functional properties and diversity of brands available in the market is increasing proportionally. This study aimed to evaluate the sensory and physical-chemical of three commercial brands available in the Brazilian market. The physical-chemical analyzes were acidity, $\mathrm{pH}$, viscosity, total and soluble solids and the sensorial evaluation were based on attribute profile and preference ranking test with untrained panelists. There was no significant difference between any of the brands evaluated for sensory properties, although they have shown significant differences in physical-chemical parameters of acidity, viscosity and soluble and total solids.
\end{abstract}

Key-words: Lactobacillus casei, probiotics, attribute profile. 


\section{Referências}

Associação Brasileira de Normas Técnicas - ABNT. Escalas utilizadas em Análise Sensorial de alimentos e bebidas. NBR 14141. Rio de Janeiro. ABNT, 1998. 3p.

Associação Brasileira de Normas Técnicas - ABNT. Teste de Ordenação em Análise Sensorial. NBR 13170. Rio de Janeiro. ABNT, 1994. 7p.

BRASIL. Ministério da Agricultura e do Abastecimento. Instrução Normativa 68 de 12 de dezembro de 2006. Métodos Analíticos Oficiais Físico-Químicos, para Controle de Leite e Produtos Lácteos. Brasília- DF, 2006.

Ministério da Agricultura e do Abastecimento. Instrução Normativa $\mathbf{n}^{\circ} 46$ de 23 de outubro de 2007. Regulamento Técnico de Identidade e Qualidade de Leites Fermentados. Brasília- DF, 2007.

CECCHI, H.M. Fundamentos teóricos e práticos em análise de alimentos. 2 ed. revisada. Campinas: Editora Unicamp, 2003. 207p.

FARIA, C. P.; BENEDET, H. D.; LE GUERROUE, J. L. Análise de leite de búfala fermentado por Lactobacillus casei e suplementado com Bifidobacterium longum. Semina: Ciências Agrárias, Londrina, v. 27, n. 3, p. 407-414, 2006.

GRANATO, D. Parâmetros físico-químicos de leites fermentados com bactérias probióticas comercializados em Curitiba (PR). Revista do Instituto de Laticínios Cândido Tostes, Juiz de Fora, n. 361, v .63, p. 39-46, 2008.

OLIVEIRA, M. N.; DARMIN, M. R. Efeito do teor de sólidos e da concentração de sacarose na acidificação, firmeza e viabilidade de bactérias do iogurte e probióticas em leite fermentado. Ciência e Tecnologia de Alimentos, Campinas, v. 23, p. 172-176, 2003. D.O.I: 10.1590/S0101-20612003000400032

ORDOÑEZ, J. A. Tecnologia de Alimentos - Volume 1: Componentes dos alimentos e processos. Porto Alegre, Artmed, 2005. 279p.

THAMER, K. G.; PENNA, A. L. B. Caracterização de bebidas lácteas funcionais fermentadas por probióticos e acrescidas de prebiótico. Ciência e Tecnologia de Alimentos, Campinas, v. 26, n. 3, p. 589-595, 2006. D.O.I:10.1590/S0101-20612006000300017

VEISSEYRE, R. Lactologia técnica - Composición, recogida, tratamiento y transformación de la leche. Editorial Acribia, Zaragoza, 1988. 629p.

VINDEROLA, C. G.; PROSELLO, W.; GHIBERTO, D.; REINHEIMER, J. A. Viability of probiotic (Bifidobacterium, Lactobacillus acidophilus and Lactobacillus casei) and nonprobiotic microflora in Argentinian fresco cheese. Journal of Dairy Science, Champaign, v. 83, p. 1905-1911, 2000. D.O.I:10.3168/jds.S0022-0302(00)75065-X

WANG, J.; GUO, Z.; ZHANG, Q.; YAN L.; CHEN, W.; LIU, X.M.; ZHANG, H.P. Fermentation characteristics and transit tolerance of probiotic Lactobacillus casei Zhang in soymilk and bovine milk during storage. Journal of Dairy Science, Champaign, v. 92, n. 6, p. 2468-76, 2009 D.O.I:10.3168/jds.2008-1849

Submetido em 26 mai 2010; Revisão recebida do revisor em 24 out. 2010; Aceito para publicação em 30 jun.2011. 